\title{
Evolution of Planetesimals in Planetary Debris Disks
}

\author{
Scott J. Kenyon \\ Smithsonian Astronomical Observatory, 60 Garden Street, Cambridge, \\ MA 02138
}

\author{
Benjamin C. Bromley \\ Department of Physics, University of Utah, $115 \mathrm{~S} 1400 \mathrm{E}, \mathrm{Rm}$ 201, Salt \\ Lake City, UT 84112
}

\begin{abstract}
Dusty planetary disks can form quickly around young stars and linger, despite the depletion of dust through mechanisms such as Poynting-Robertson drag. Planetesimals in the disk can replenish the dust with an efficiency that depends on their mass and velocity distributions. Here, we describe a numerical program to track the evolution of planetesimals through merging, fragmentation and drag. Our current effort is focused on coupling a Fokker-Planck solver with N-body calculations to follow the detailed behavior of larger planetesimals. Our results demonstrate the importance of gravitational stirring in dust production. Furthermore, stirring by planets larger than Pluto can generate the large scale heights seen in several dusty disks.
\end{abstract}

\section{Introduction}

We now see from direct observation that planetary debris disks form around nearly all young, main sequence stars. Studies in wavebands ranging from the far-infrared to optical suggest that debris disks have ring-like structures composed of small dust grains (e.g., Trilling \& Brown 1998). Furthermore, they extend to several hundred AU, even to $1000 \mathrm{AU}$ in some cases, and show a small hole near the central star which is dust-free. Surveys from the Infrared Space Observatory indicate a mean life-time for planetary debris disks of 400 million years (e.g., Becklin et al. 1998). Debris disks may contain as much as 0.1 earth masses $\left(M_{E}\right)$ with a low gas content; typical gas-to-dust mass ratios range from 1 to 10 (e.g., Zuckerman et al. 1995).

There are several mechanisms which tend to clear both gas and dust from around a young star, including radiation pressure, Poynting-Robertson drag, and solar wind. As a result planetary debris disk easily could be swept away within a 1 Myr. Thus, there must be a reservoir of matter that can replenish the dust. A popular kodel for the replenishment of dust is that the debris disk contains up to $100 M_{E}$ in the form of planetesimals, similar to those found in the Kuiper Belt around the Sun. Planetesimals can release dust in a "collisional cascade" whereby collisions between two large planetesimals produce more numerous, smaller planetesimals, which themselves collide. The viability of this model 
depends critically on generating the necessary high collision velocities (100$300 \mathrm{~km} / \mathrm{s}$ ) to produce such a cascade.

Here we present calculations to determine the role of gravitational stirring by larger planetesimals as initiators of the collisional cascade. We model the evolution of a planetary debris disk using a Fokker-Plank method (see Kenyon \& Luu 1998, also Kenyon \& Bromley 2001) with both single-mass planetesimals and planetesimals with a broad mass distribution. The system is evolved according to the Fokker-Planck equation to account for viscous stirring and dynamical friction. We introduce gravitational stirring with one or two populations of larger planetesimals of fixed mass. Standard assumptions about the ejected material from a two-body icy planetesimal collision, in conjunction with disk parameters (e.g., velocity distribution, scale height) then yield estimates of the dust production rate and disk lifetime.

\section{Results}

We ran our code on a parallel supercomputer to evolve planetary debris disks. Tests indicate that the code output matches the results of previous work (see Stewart \& Ida 2000). Our main result is that a range of planetesimal mass is needed in order to gravitationally stir a debris disk. A single population of planetesimals, even with radii as large as $100 \mathrm{~km}$, can not produce velocities higher than $100 \mathrm{~km} / \mathrm{s}$ in 1 Gyr. For a distribution with constant mass in logarithmic mass bins, planetesimals as large as $500 \mathrm{~km}$ in radius are essential for generating high collision velocities in the disk. We also find that once a collisional cascade is initiated, the scale height can become comparable to the values inferred from observations if large planetesimals are present in the planetary debris disk. Finally, our results show that the total disk mass must be comparable to the minimum mass solar nebula in order to maintain a collisional cascade beyond a few hundred Myr.

Our code currently can track fragmentation, gas and Poynting-Robertson drag, and dynamical friction, as well as viscous and gravitational stirring. We are now in the process of implementing an N-body component (Ida \& Makino 1993) to more directly model the effects of large planetesimals and planets.

We acknowledge a generous allocation on the SGI Origin-2000 at JPL Supercomputing, funded by NASA Offices of Mission to Planet Earth, Aeronautics, and Space Science.

\section{References}

Becklin, E., E., et al. 1998, Ap\&SS, 255, 113

Ida, S., \& Makino, J. 1993, Icarus, 106, 210

Kenyon, S. J., \& Bromley, B. C. 2000, AJ, 121, 538

Kenyon, S. J., \& Luu, J. X. 1998, AJ, 115, 2136

Stewart, G. R., \& Ida, S. 2000, Icarus, 143, 28

Trilling, D. E., \& Brown, R. H. 1998, Nature, 395, 775

Zuckerman, B., Forveille, T., \& Kastner, J. H. 1995, Nature, 373, 494 